\title{
PIM1 knockdown inhibits cell proliferation and invasion of mesothelioma cells
}

\author{
AMANY SAYED MAWAS ${ }^{1,2^{*}}$, VISHWA JEET AMATYA $^{1 *}$, RUI SUZUKI $^{1,3}$, \\ KEI KUSHITANI $^{1}$, MOUCHIRA M. MOHI EL-DIN ${ }^{2}$ and YUKIO TAKESHIMA ${ }^{1}$ \\ ${ }^{1}$ Department of Pathology, Institute of Biomedical and Health Sciences, Hiroshima University, Hiroshima, Japan; \\ ${ }^{2}$ Department of Pathology and Clinical Pathology, Faculty of Veterinary Medicine, South Valley University, \\ Qena, Egypt; ${ }^{3}$ Hiroshima University School of Medicine, Hiroshima, Japan
}

Received December 5, 2016; Accepted January 20, 2017

DOI: $10.3892 /$ ijo.2017.3863

\begin{abstract}
Malignant mesothelioma is a major asbestos-related cancer with prolonged time lapse from the first exposure of asbestos to the development of mesothelioma. Most of mesothelioma patients show very poor prognosis, thus, an urgent improvement of its treatment is required by development of novel therapeutic strategies. RNA interference (RNAi) is a powerful tool in post-genomic research and cancer therapy through inhibition of gene expression. In the present study, we analyzed the function of PIM1 on mesothelioma cell lines with its knockdown by siRNA transfection. Here, we report that the downregulation of PIM1 led to suppression of cell proliferation by cell cycle arrest at G1 phase and suppression of cell invasion and migration. Considering the mesothelioma as rapidly growing invasive cancer, downregulation of PIM1 may have a potential role for therapeutic management of malignant mesothelioma.
\end{abstract}

\section{Introduction}

Malignant mesothelioma, highly aggressive fatal cancer, is associated with occupational and environmental asbestos exposure (1). Its incidence is increasing in Japan, Western European countries and is expected to increase in other developing countries (2). The molecular mechanism of its carcinogenesis is not yet fully understood. Previously, we studied microRNA expression profile of mesothelioma cell lines and found that significant numbers of microRNA were differentially expressed

Correspondence to: Professor Yukio Takeshima, Department of Pathology, Institute of Biomedical and Health Sciences, Hiroshima University, 1-2-3 Kasumi, Minami-ku, Hiroshima 734-8551, Japan E-mail: ykotake@hiroshima-u.ac.jp

*Contributed equally

Key words: mesothelioma cell line, PIM1, proliferation, invasion, siRNA in malignant mesothelioma. In addition to various upregulated microRNAs in mesothelioma cell lines, we found no expression of many microRNAs, namely miR-1 and miR-214. By microRNA mimic transfection study, we reported the role of miR-1, and miR-214 in proliferation and invasion of mesothelioma cells. Many genes are co-targeted by miR-1 and miR-214. Out of these genes, we paid attention to PIM1 gene, a proto-oncogene, for its high expression in mesothelioma cell lines (3). Provirus integration site for Moloney murine leukemia virus 1 (PIM1) is a serine/threonine kinase that acts as protooncogene to mediate cell survival. PIM1 has been reported to be upregulated in various human cancers such as prostate (4), pancreatic (5), gastric cancer (6) and glioblastoma (7). The overexpression of PIM1 was well correlated to carcinogenesis by promoting tumor cell proliferation and inhibiting apoptosis (8). In the present study, we analyzed the proliferation, the cell cycle regulation, invasion and the migration of mesothelioma cells by PIM1 downregulation with siRNA transfection.

\section{Materials and methods}

Mesothelioma cell lines. Mesothelioma cell lines, CRL-5915 (purchased from the American Type Culture Collection, ATCC; Manassas, VA, USA) and ACC-MESO-1 (RIKEN BioResearch Center, Tsukuba, Japan) (9) were maintained in Roswell Park Memorial Institute 1640 medium with GlutaMAX (RPMI-1640) with 1\% kanamycin, 1\% fungizone and $10 \%$ fetal bovine serum (FBS; all purchased from Gibco/ Life Technologies, Tokyo, Japan).

Transient transfections of mesothelioma cells with small interfering RNA (siRNA) after siRNA optimization. To optimize $\mathrm{IC}_{50}$ (half-maximum inhibitory concentration) of siRNA, we used different concentrations of siRNA for both cell lines which not causing cytotoxic effect on mesothelioma cell lines (20, 30 and $40 \mathrm{nM})$. The cells were cultured $3 \times 10^{3}$ with the different concentrations of siRNA in 96-well plate for three days then adding PrestoBlue reagent (PrestoBlue ${ }^{\mathrm{TM}}$ Cell Viability reagent; Invitrogen) every day and cell viability was measured by GloMax ${ }^{\circledR}$ Explorer. All transfection studies were carried out in triplicate with $20 \mathrm{nM}$ of PIM1-siRNA or negative control-siRNA with $5 \mu$ l of Lipofectamine RNAiMax 
reagent in Opti-MEM media (Gibco) in 6-well plates for $24 \mathrm{~h}$ before harvesting the treated cells. Details of PIM1-siRNA were (siGENOME SMARTpool PIM-siRNA, GAUAUGGU GUGUGGAGAUA, CAGAUAGGCCAACCUUCGA, GUG GAGAAGGACCGGAUUU, AGAUAUUCCUUUCGAGC AU) and negative control-siRNA (UAGCGACUAAACAC AUCAA) (Dharmacon, Lafayette, CO, USA). After transfection with siRNAs, the cells were utilized for various cell based assays, cell proliferation, cell cycle, invasion and migration assays.

RNA isolation. Total RNA was extracted from the mesothelioma cell lines using CellAmp ${ }^{\mathrm{TM}}$ Direct RNA Prep kit (Takara/Clontech Laboratories) according to the manufacturer's recommended protocol. The extracted RNA was quantified with a NanoVue Plus spectrophotometer (GE Healthcare BioSciences, Tokyo, Japan) and Qubit 2.0 (Life Technologies) using fluorometer-based RNA assay kit.

Real-time reverse transcription polymerase chain reaction. A total of $200 \mathrm{ng}$ of total RNA was reverse-transcribed and amplified using One Step SYBR ${ }^{\circledR}$ PrimeScript ${ }^{\circledR}$ RT-PCR kit (Takara Bio) with the Mx3000P real-time PCR (Stratagene, La Jolla, CA, USA) system. Relative expression was calculated by the comparative CT $(\Delta \Delta \mathrm{CT})$ method.

Western blot analysis. Cells $\left(5 \times 10^{5}\right)$ were seeded and transfected in 6-well plates for 3 days and cell lysates were obtained from siRNA transfected cells using M-PER (mammalian protein extraction reagent) with Halt Protease Inhibitor Cocktail and Phosphatase inhibitor (Thermo Fisher Scientific, Waltham, MA, USA). Total protein (20-30 $\mu \mathrm{g}$ ) was electrophoresed on TGX acrylamide Gel (Bio-Rad Laboratories, Tokyo, Japan) at $75 \mathrm{~V}$ for $90 \mathrm{~min}$ and transferred onto a polyvinylidene difluoride (PVDF) membrane using Mini Blot Module (Thermo Fisher Scientific) at $20 \mathrm{~V}$ for $60 \mathrm{~min}$. The protein-transferred membrane was processed with primary antibodies, anti-PIM1 antibody (1:2000, rabbit monoclonal, ab75776; Abcam) and anti-GAPDH antibody (1:1000, rabbit polyclonal sc-25778; Santa Cruz Biotechnology, Dallas, TX, USA) and biotin labeled secondary antibody (HRP-linked goat anti-rabbit IgG, 7074S; Cell Signaling Technology, Danvers, MA, USA) and all were diluted in Can Get Signal buffer (Toyobo Life Science, Osaka, Japan). The membrane was washed and stained with ImmunoCruz ${ }^{\mathrm{TM}}$ Western Blotting Luminol reagent (Santa Cruz Biotechnology) and images were captured using C-DiGit Blot scanner (LI-COR Biosciences, Lincoln, NE, USA) with Image Studio software.

Cell proliferation assay. siRNA transfected cells $\left(3 \times 10^{3}\right)$ were incubated in RPMI-1640 media with 1\% FBS in 96-well plates in triplicate for 3 days. Total number of viable cells (based on quantitation of ATP present, which indicate the presence of metabolically active cells) were determined every 24,48 and $72 \mathrm{~h}$ by CellTiter-Glo 2.0 reagent (Promega) according to the manufacturer's recommended protocol using GloMax Explorer.

Cell cycle phase analysis. siRNA transfected cells $\left(5 \times 10^{4}\right)$ were incubated in RPMI-1640 media with 1\% FBS in collagen-coated 12-well plates in triplicate for 48 and $72 \mathrm{~h}$. Cells were harvested after trypsinization and slowly fixed in $70 \%$ ethanol for more than $1 \mathrm{~h}$. Ethanol fixed cells were washed and stained with Guava Cell Cycle reagent and data were collected with flow cytometer using Cytosoft cell cycle module software (Guava Technologies, Hayward, CA, USA). Guava EasyCyte Mini flow cytometric raw data were analyzed using FCS Express 5 Pro software (De Novo Software, Glendale, CA, USA).

Cell invasion assay. siRNA transfected cells $\left(1 \times 10^{4}\right)$ for ACC-MESO-1 and $1.5 \times 10^{5}$ siRNA transfected cells for CRL-5915 were incubated in BD FluoroBlok culture inserts with $8-\mu \mathrm{m}$ pores (BD Biosciences, Franklin Lakes, NJ, USA) coated with matrix Matrigel (Life Technologies) according to the manufacturer's protocol. Invaded cells were stained with Hoechst 33342 (Life Technologies) and imaged area of insert membrane was captured with fluorescent microscope (IX81, inverted microscope with DP80 CCD camera; Olympus, Tokyo, Japan). The total numbers of invading cells were calculated by analyzing fluorescent images with CellProfiler cell imaging software (10).

Cell migration assay. Cell migration was analyzed using a wound/scratch assay. siRNA transfected cells were incubated in RPMI-1640 media with 10\% FBS in collagen-coated 24-well plates overnight. The wound was created in cell monolayer by scratching the cells with $1 \mathrm{ml}$ micropipette tips and floating cells were removed by washing with fresh media. Real-time microscopic pictures were taken at 0,12 and $24 \mathrm{~h}$ post scratching by incubating the cells in stage-top incubator on inverted microscope with automatic picture acquisition at given time interval (Olympus IX81 with cellSens software). The area of the wound gap that was not covered by cell migration (percentage) was determined using TScrach software (11).

Statistical analysis. All statistical analysis was performed with GraphPad QuickCalcs t-test calculator (https://www. graphpad.com/quickcalcs/).

\section{Results}

Validation of PIM1 knockdown by siRNA transfection. After optimization of $\mathrm{IC}_{50}$ of siRNA, we found that all the different concentration of PIM1-siRNA and negative control-siRNA have less cytotoxic effect on both cell lines. With $20 \mathrm{nM}$ of PIM1-siRNA, we found that the downregulation of PIM1 mRNA was from 50 to $70 \%$ (Fig. 1A). Significant downregulation of PIM1 was found at protein level by western blot analysis (Fig. 1B).

PIM1 knockdown reduces cell proliferation with G1 arrest. Mesothelioma cell lines transfected with PIM1-siRNA showed $\sim 20 \%$ reduced proliferation rate compared to that with negative control-siRNA in a time-dependent manner (Fig. 2 and Table I). In addition, we also noted difference in the proliferation rates between cell lines.

With siRNA transfection of mesothelioma cells for 2 days, the proportion of G1 phase of cell cycle in ACC-MESO-1 and CRL-5915 with PIM1 transfection were 60.5 and $65.8 \%$ 
Table I. Cell proliferation analysis.

$0 \mathrm{~h} \quad 24 \mathrm{~h} \quad 48 \mathrm{~h} \quad 72 \mathrm{~h}$

\begin{tabular}{lllll}
\hline CRL-5915 & & & & \\
PIM1 siRNA & $1.04 \pm 0.10$ & $1.35 \pm 0.10$ & $2.27 \pm 0.10$ & $3.17 \pm 0.10$ \\
Negative control siRNA & $1.25 \pm 0.03$ & $1.89 \pm 0.10$ & $3.13 \pm 0.14$ & \\
ACC-MESO-1 & & & & \\
PIM1 siRNA & $1.81 \pm 0.11$ & $2.14 \pm 0.17$ & $2.88 \pm 0.11$ & $3.29 \pm 0.33$ \\
Negative control siRNA & $2.02 \pm 0.12$ & $2.44 \pm 0.10$ & $3.47 \pm 0.11$ & $4.70 \pm 0.03$ \\
\hline
\end{tabular}

The number of proliferating cells every $24 \mathrm{~h}$ is significantly less in PIM1 siRNA transfected cells compared to the negative control siRNA transfected cells showing inhibition of proliferation rate (see Fig. 2).

Table II. Cell cycle phase analysis.

G1 $\quad$ S $\quad$ G2 $\quad$ P-value

\begin{tabular}{llll}
\hline ACC-MESO-1 & & & \\
PIM1 siRNA & $60.5 \pm 1.0$ & $20.6 \pm 0.6$ & $20.6 \pm 1.0$ \\
Negative control siRNA & $43.3 \pm 1.2$ & $32.8 \pm 5.4$ & $32.8 \pm 5.0$ \\
CRL-5915 & & & 0.0001 \\
PIM1 siRNA & $65.8 \pm 2.7$ & $19.5 \pm 2.6$ & $14.7 \pm 1.5$ \\
Negative control siRNA & $50.0 \pm 1.4$ & $25.2 \pm 0.9$ & $24.7 \pm 1.6$
\end{tabular}

The percentage of G1 phase of cell cycle in PIM1 siRNA transfected cells is significantly higher than that in negative control siRNA transfected cells in both ACC-MESO-1 and CRL-5915 cell lines (see Fig. 3).
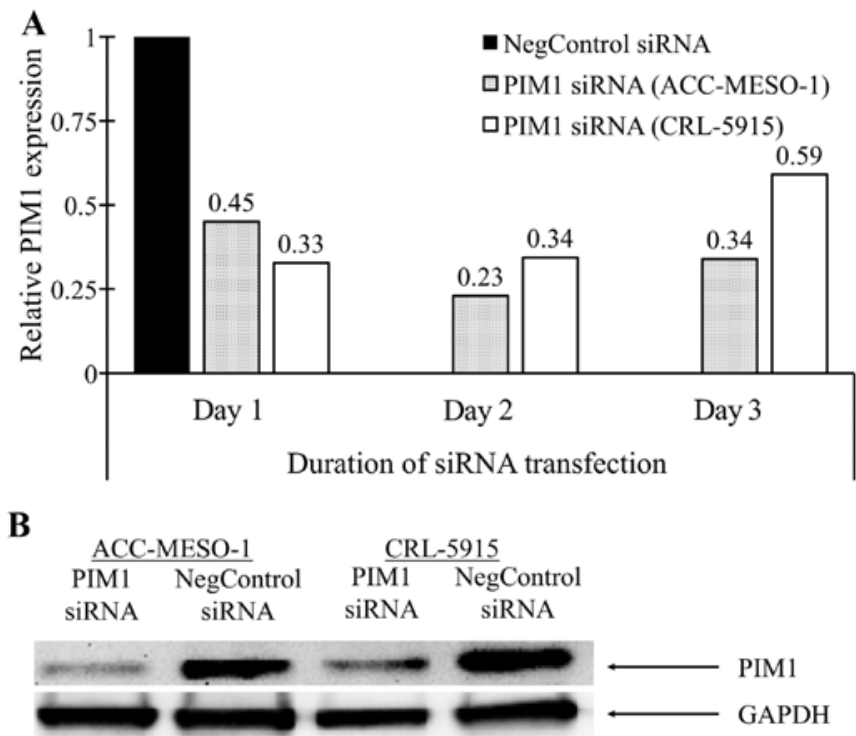

Figure 1. (A) PIM1 expression by RT-PCR. Downregulation of PIM1 expression by $50-75 \%$ in PIM1-siRNA transfected cells compared to negative control-siRNA (NC-siRNA) in mesothelioma cell lines, ACC-MESO-1 and CRL-5915. (B) PIM1 expression by western blot analysis. Downregulation of PIM1 protein expression in PIM-siRNA transfected cells for 2 days compared to NC-siRNA.

and that with negative control was 43.3 and $50 \%$ (Fig. 3 and Table II). With siRNA transfection of mesothelioma

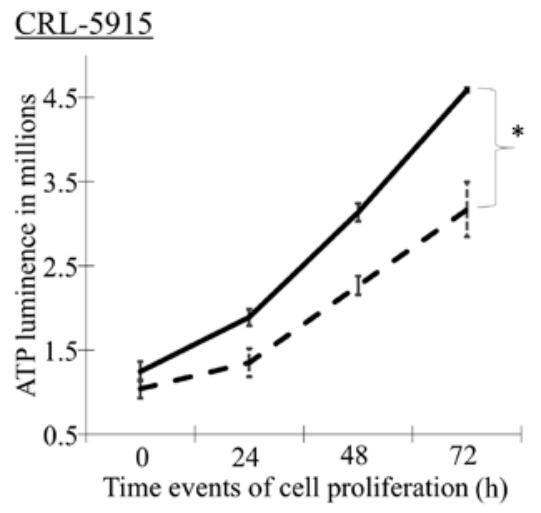

\section{$\underline{\text { ACC-MESO-1 }}$}

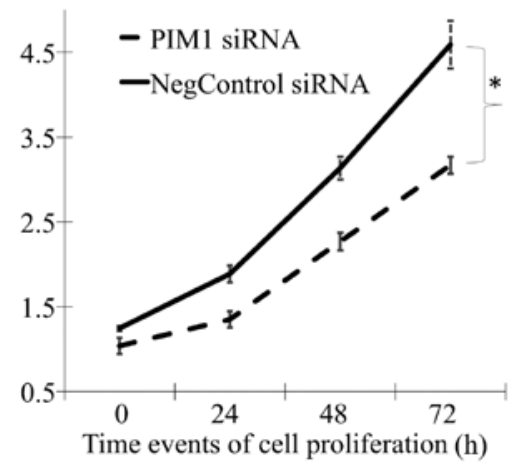

Figure 2. Proliferation assay. ACC-MESO-1 and CRL-5915 cells show lower proliferation rate in PIM1-siRNA transfected cells than NC-siRNA transfected cells in time based manner $(24,48$ and $72 \mathrm{~h})(\mathrm{P}<0.01)$. 

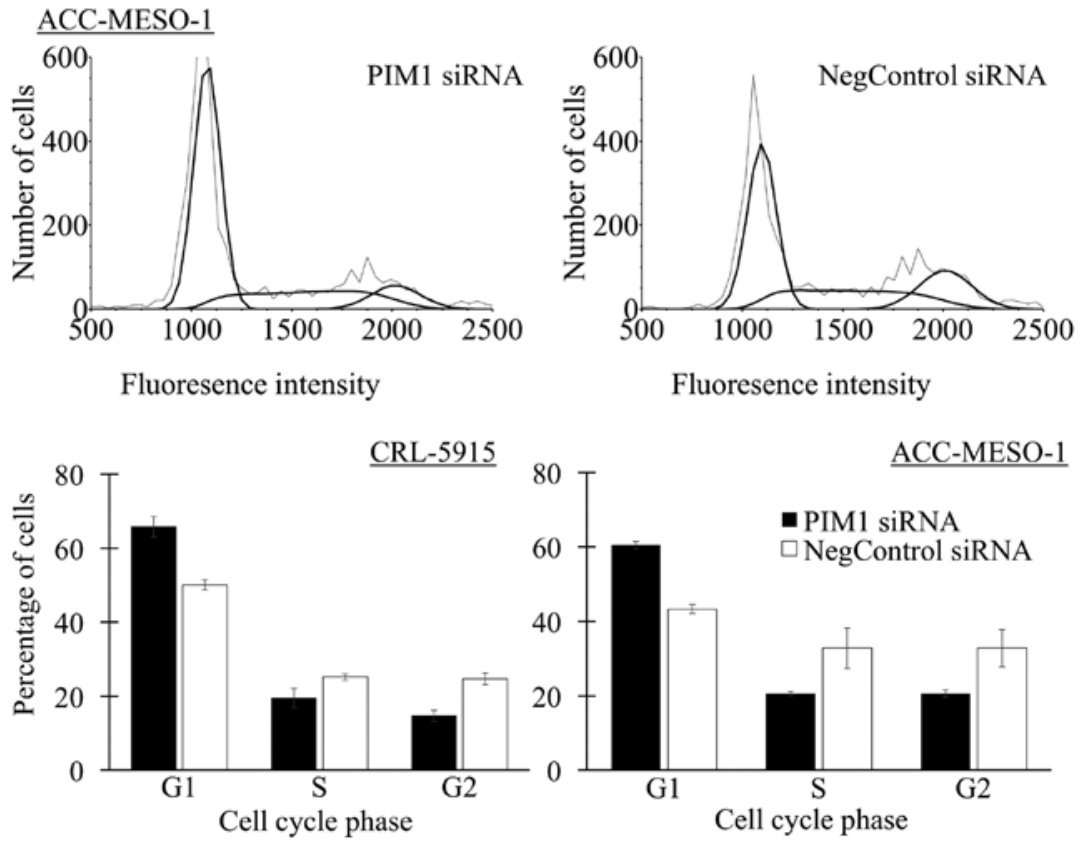

Figure 3. Cell cycle assay. ACC-MESO-1 and CRL-5915 cells show increase in G1 phase cells in PIM1-siRNA transfected cells compared to NC-siRNA transfected cells after $48 \mathrm{~h}$.

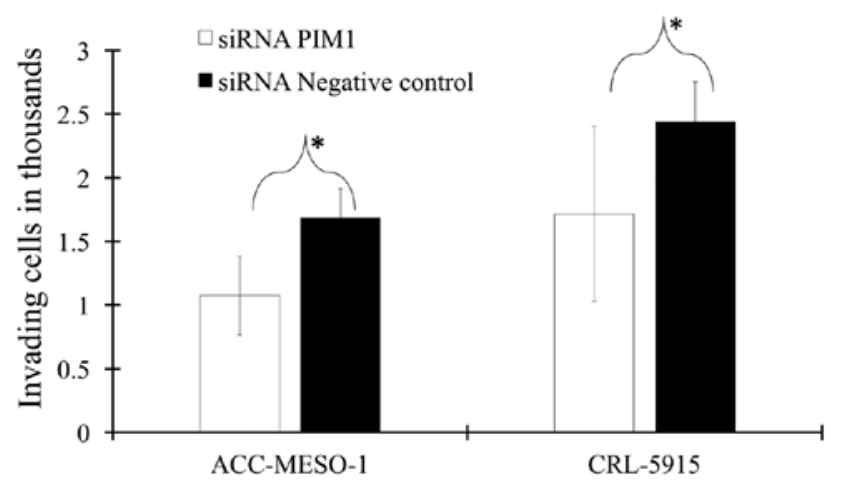

Figure 4. Invasion assay. ACC-MESO-1 and CRL-5915 cells transfected with PIM1-siRNA for two days show less invasion ( $\sim 30 \%$ less invasive) during 2 days of incubation compared to NC-siRNA transfected cells through the Matrigel layer.

cells for 3 days, the proportion of G1 phase of cell cycle in ACC-MESO-1 and CRL-5915 with PIM1 transfection were 84 and $78.6 \%$ and that with negative control was 74.5 and $74.1 \%$.

These results suggest that PIM1-siRNA transfected cells showed $\sim 10 \%$ increase in G1 phase compared to negative control-siRNA. In addition, the ratio of G1 arrest was higher on day 2 than day 3 in both cell lines.

PIM1 knockdown reduces cell invasion and cell migration. We found that the ability of ACC-MESO-1 for invasion is faster than CRL-5915 as ACC-MESO-1 invade via the Matrigel layer in 2 days while CRL-5915 need 3-4 days for its invasion, thus, we used reduced number of cells and increased thickness of the Matrigel layer in case of ACC-MESO-1.

Mesothelioma cells with PIM1 transfection showed 30\% less invasiveness compared to that with negative control-siRNA transfection (Fig. 4 and Table III). Similarly, the mesothelioma
Table III. Invasion assay.

\begin{tabular}{lccc}
\hline & \multicolumn{3}{c}{$\begin{array}{c}\text { No. of invading cells/ one } \\
\text { quadrant of insert }\end{array}$} \\
\cline { 2 - 4 } & Mean & SD & P-value \\
\hline CRL-5915 & & & \\
PIM1 siRNA & 1716 & 313 & 0.0168 \\
Negative control siRNA & 2439 & 686 & \\
ACC-MESO-1 & & & \\
PIM1 siRNA & 1074 & 230 & 0.0005 \\
Negative control siRNA & 1684 & 309 & \\
\hline
\end{tabular}

The difference in the ability of cells to invade through the Matrigel indicating that invading number of PIM1 siRNA transfected cells is less than negative control siRNA in ACC-MESO-1 and CRL-5915 cell lines (see Fig. 4).

cells with PIM1 transfection showed less migration than that with negative control-siRNA (Fig. 5).

\section{Discussion}

The conventional management of mesothelioma by either surgery or radiotherapy and chemotherapy are not very effective, and even the recent clinical trials of trimodal therapy with neoadjuvant chemotherapy followed by extra-pleural pneumonectomy and radiotherapy have not shown significant advantage over conventional therapy (12-14). In populationbased studies, survival times ranged from 5 months to 13.2 months (15). Although surgery is an option for patients with early-stage mesothelioma, most patients present with advanced locally invasive disease, not amenable to surgical resection. 
A
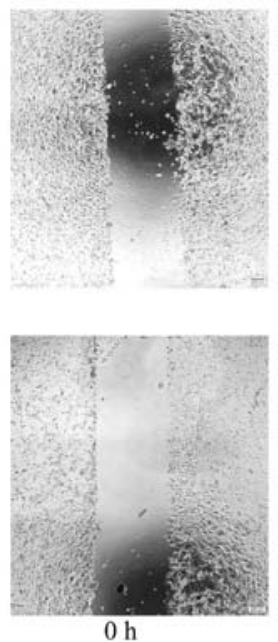

$\mathrm{Oh}$
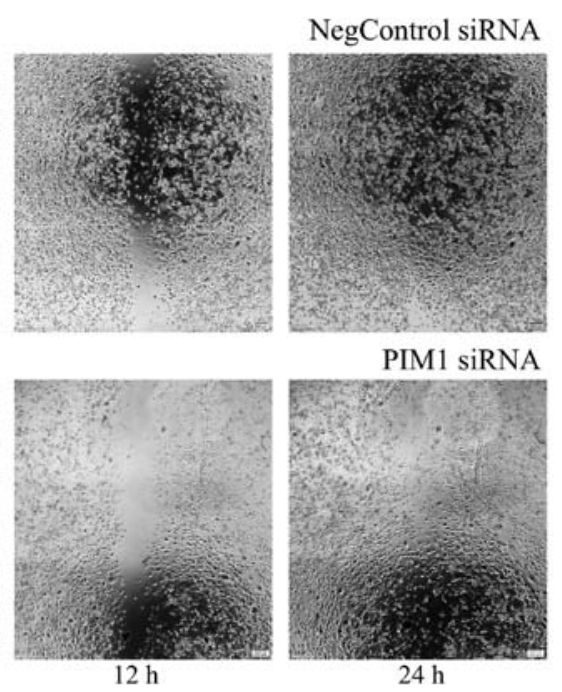

B

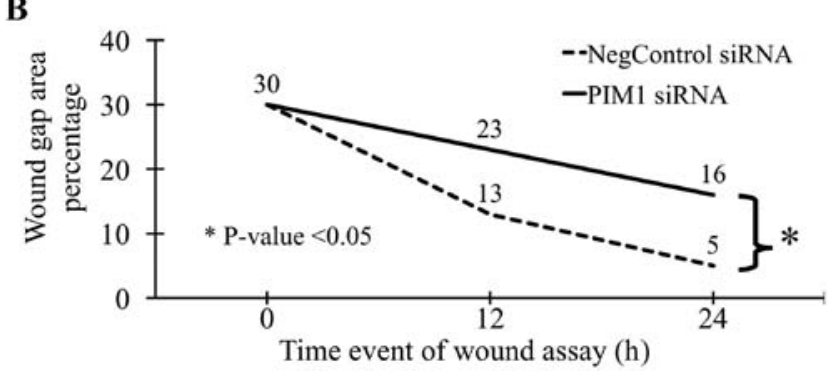

Figure 5. Migration assay. ACC-MESO-1 and CRL-5915 cells transfected with PIM1-siRNA show less migration (decreased wound gap closure) during 24-h incubation compared to NC-siRNA transfected cells $\left({ }^{*} \mathrm{P}<0.05\right)$.

For these patients, the current best regime is palliative chemotherapy with cisplatin and pemetrexed. However, this regimen has been shown a modest survival benefit (16). Therefore, the novel strategies for management of mesothelioma patients have to be developed urgently in the coming years. There are various trials ongoing for the treatment of malignant mesothelioma. A recent study has previously reported the high expression and prognostic value of CD26 in malignant mesothelioma $(17,18)$, humanized anti-CD26 monoclonal antibody, YS-110, having inhibitory effect on malignant mesothelioma cell growth in vitro and in vivo experiments (19), and one of the promising new developments for the antibody-based treatment of mesothelioma $(19,20)$. Other trials such as pembrolizumab against programmed death 1 (PD-1) antibody, PF-03446962 against activin receptor-like kinase 1 (ALK-1), defactinib against focal adhesion kinase (FAK) are ongoing (21-23).

RNA interference (RNAi) has broad potential as a therapeutic role to reversibly silence a gene. Recently delivery of siRNA to the targeted tissue has been improved to achieve the clinical potential of RNAi $(24,25)$. We have previously reported the high expression of nuclear PIM1 in mesothelioma by immunohistochemical staining and mesothelioma cell lines by western blot analysis (3). Upregulation of PIM1 has been reported in prostate (4), pancreatic (5) and non-small cell lung cancers (6) with good prognostic significance. However, its high expression in stomach (6), bladder (26), esophagus (26) and head and neck carcinoma (27) was correlated with poor prognosis. Therefore, PIM1 may have different functions in tumorigenesis depending on the tumor type and underlying signaling pathways. We investigated the functional role of PIM1 in mesothelioma cell lines by siRNA-based knockdown and confirmed its potential role on cell survival, migration and invasion. PIM1 overexpression has been shown to play a major role in the inhibition of apoptosis and the regulation of cell proliferation (28) as well as in promotion of cell survival through phosphorylation of other substrate proteins as a proapoptotic factor FOXO3a (8). The level of PIM1 has been shown to increase during the progression of the cell cycle from G1 and remain high throughout G1/S and G2 phases, thus, PIM1 protein level changes during cell cycle stages appear to be related to the onset of DNA synthesis and commitment to proliferation (29) and high level of PIM1 activate cell cycle inhibitors as p21 and p27 thereby promote cell cycle phases (30). We found that the downregulation of PIM1 caused decrease in proliferation rates of mesothelioma cells and cell cycle analysis have shown the accumulation of mesothelioma cells in G1 phase. We also analyzed the potential role of PIM1 to influence the invasion of mesothelioma cell lines by Transwell invasion assay and wound assay and found significant decrease in invasion through Matrigel and decrease in migration of mesothelioma cells. Based on our preliminary results, PIM1 expression in human mesothelioma tissue may be useful in differential diagnosis of malignant mesothelioma from the reactive mesothelial hyperplasia (data not shown).

Increasing evidence has shown that PIM1 would be a novel and essential drug target in numerous types of cancer (31), in particular prostate cancer (4). Novel molecules inhibiting PIM kinases have been evaluated in preclinical studies, demonstrating to be effective and with a favorable toxicity profile. Given the promising results, some of these compounds are currently under investigation in clinical trials (32). We analyzed in this study the possibility for application of therapeutic potential in mesothelioma.

In conclusion, the downregulation of PIM1 in mesothelioma cell have shown to reduce the proliferation, invasion and migration of mesothelioma cells. Considering that mesothelioma is a rapidly growing invasive cancer, downregulation of PIM1 has potential for therapeutic management of mesothelioma. However, further mechanism of regulation of its downstream genes is needed to understand how its regulation modulates the mesothelioma cells.

\section{Acknowledgements}

The authors thank Ms. Naomi Fukuhara for her administrative assistance. Part of the present study was carried out at the Analysis Center of Life Science, Hiroshima University.

\section{References}

1. Delgermaa V, Takahashi K, Park EK, Le GV, Hara T and Sorahan T: Global mesothelioma deaths reported to the World Health Organization between 1994 and 2008. Bull World Health Organ 89: 716-724, 724A-724C, 2011.

2. McDonald JC and McDonald AD: The epidemiology of mesothelioma in historical context. Eur Respir J 9: 1932-1942, 1996.

3. Amatya VJ, Mawas AS, Kushitani K, Mohi El-Din MM and Takeshima Y: Differential microRNA expression profiling of mesothelioma and expression analysis of miR-1 and miR-214 in mesothelioma. Int J Oncol 48: 1599-1607, 2016. 
4. Dhanasekaran SM, Barrette TR, Ghosh D, Shah R, Varambally S, Kurachi K, Pienta KJ, Rubin MA and Chinnaiyan AM: Delineation of prognostic biomarkers in prostate cancer. Nature 412: 822-826, 2001.

5. Reiser-Erkan C, Erkan M, Pan Z, Bekasi S, Giese NA, Streit S, Michalski CW, Friess $\mathrm{H}$ and Kleeff J: Hypoxia-inducible protooncogene Pim-1 is a prognostic marker in pancreatic ductal adenocarcinoma. Cancer Biol Ther 7: 1352-1359, 2008.

6. Warnecke-Eberz U, Bollschweiler E, Drebber U, Metzger R Baldus SE, Hölscher AH and Mönig S: Prognostic impact of protein overexpression of the proto-oncogene PIM-1 in gastric cancer. Anticancer Res 29: 4451-4455, 2009.

7. Herzog S, Fink MA, Weitmann K, Friedel C, Hadlich S, Langner S, Kindermann K, Holm T, Böhm A, Eskilsson E, et al: Pim1 kinase is upregulated in glioblastoma multiforme and mediates tumor cell survival. Neuro Oncol 17: 223-242, 2015.

8. Magnuson NS, Wang Z, Ding G and Reeves R: Why target PIM1 for cancer diagnosis and treatment? Future Oncol 6: 1461-1478, 2010.

9. Usami N, Fukui T, Kondo M, Taniguchi T, Yokoyama T, Mori S, Yokoi K, Horio Y, Shimokata K, Sekido Y, et al: Establishment and characterization of four malignant pleural mesothelioma cell lines from Japanese patients. Cancer Sci 97: 387-394, 2006.

10. Lamprecht MR, Sabatini DM and Carpenter AE: CellProfiler: Free, versatile software for automated biological image analysis. Biotechniques 42: 71-75, 2007.

11. Gebäck T, Schulz MM, Koumoutsakos P and Detmar M: TScratch: A novel and simple software tool for automated analysis of monolayer wound healing assays. Biotechniques 46 : 265-274, 2009.

12. Hasegawa S, Okada M, Tanaka F, Yamanaka T, Soejima T, Kamikonya N, Tsujimura T, Fukuoka K, Yokoi K and Nakano T: Trimodality strategy for treating malignant pleural mesothelioma: Results of a feasibility study of induction pemetrexed plus cisplatin followed by extrapleural pneumonectomy and postoperative hemithoracic radiation (Japan Mesothelioma Interest Group 0601 Trial). Int J Clin Oncol 21: 523-530, 2016.

13. Kapeles M, Gensheimer MF, Mart DA, Sottero TL, Kusano AS Truong A, Farjah F, Laramore GE, Stelzer KJ and Patel SA: Trimodality treatment of malignant pleural mesothelioma: An Institutional Review. Am J Clin Oncol: Aug 27, 2015 (Epub ahead of print).

14. Krug LM, Pass HI, Rusch VW, Kindler HL, Sugarbaker DJ, Rosenzweig KE, Flores R, Friedberg JS, Pisters K, Monberg M, et al: Multicenter phase II trial of neoadjuvant pemetrexed plus cisplatin followed by extrapleural pneumonectomy and radiation for malignant pleural mesothelioma. J Clin Oncol 27: 3007-3013, 2009

15. Montanaro F, Rosato R, Gangemi M, Roberti S, Ricceri F, Merler E, Gennaro V, Romanelli A, Chellini E, Pascucci C, et al: Survival of pleural malignant mesothelioma in Italy: A population-based study. Int J Cancer 124: 201-207, 2009.

16. Marinaccio A, Montanaro F, Mastrantonio M, Uccelli R, Altavista P, Nesti M, Costantini AS and Gorini G: Predictions of mortality from pleural mesothelioma in Italy: A model based on asbestos consumption figures supports results from age-periodcohort models. Int J Cancer 115: 142-147, 2005.

17. Amatya VJ, Takeshima Y, Kushitani K, Yamada T, Morimoto C and Inai K: Overexpression of CD26/DPPIV in mesothelioma tissue and mesothelioma cell lines. Oncol Rep 26: 1369-1375, 2011.
18. Aoe K, Amatya VJ, Fujimoto N, Ohnuma K, Hosono O, Hiraki A, Fujii M, Yamada T, Dang NH, Takeshima Y, et al: CD26 overexpression is associated with prolonged survival and enhanced chemosensitivity in malignant pleural mesothelioma. Clin Cancer Res 18: 1447-1456, 2012.

19. Inamoto $\mathrm{T}$, Yamada $\mathrm{T}$, Ohnuma K, Kina S, Takahashi $\mathrm{N}$, Yamochi T, Inamoto S, Katsuoka Y, Hosono O, Tanaka H, et al: Humanized anti-CD26 monoclonal antibody as a treatment for malignant mesothelioma tumors. Clin Cancer Res 13: 4191-4200, 2007.

20. Thompson MA, Ohnuma K, Abe M, Morimoto C and Dang NH: $\mathrm{CD} 26 /$ dipeptidyl peptidase IV as a novel therapeutic target for cancer and immune disorders. Mini Rev Med Chem 7: 253-273, 2007.

21. Bickel A, Koneth I, Enzler-Tschudy A, Neuweiler J, Flatz L and Früh M: Pembrolizumab-associated minimal change disease in a patient with malignant pleural mesothelioma. BMC Cancer 16: 656, 2016.

22. Shimizu T, Fukuoka K, Takeda M, Iwasa T, Yoshida T, Horobin J, Keegan M, Vaickus L, Chavan A, Padval M, et al: A first-in-Asian phase 1 study to evaluate safety, pharmacokinetics and clinical activity of VS-6063, a focal adhesion kinase (FAK) inhibitor in Japanese patients with advanced solid tumors. Cancer Chemother Pharmacol 77: 997-1003, 2016.

23. Wheatley-Price P, Chu Q, Bonomi M, Seely J, Gupta A, Goss G, Hilton J, Feld R, Lee CW, Goffin JR, et al: A Phase II study of PF-03446962 in patients with advanced malignant pleural mesothelioma. CCTG Trial IND.207. J Thorac Oncol 11: 2018-2021, 2016.

24. Zhao $\mathrm{J}$ and Feng SS: Nanocarriers for delivery of siRNA and co-delivery of siRNA and other therapeutic agents. Nanomedicine (Lond) 10: 2199-2228, 2015.

25. Kanasty R, Dorkin JR, Vegas A and Anderson D: Delivery materials for siRNA therapeutics. Nat Mater 12: 967-977, 2013.

26. Guo S, Mao X, Chen J, Huang B, Jin C, Xu Z and Qiu S: Overexpression of Pim-1 in bladder cancer. J Exp Clin Cancer Res 29: 161, 2010.

27. Peltola K, Hollmen M, Maula SM, Rainio E, Ristamäki R, Luukkaa M, Sandholm J, Sundvall M, Elenius K, Koskinen PJ, et al: Pim-1 kinase expression predicts radiation response in squamocellular carcinoma of head and neck and is under the control of epidermal growth factor receptor. Neoplasia 11: 629-636, 2009

28. Amaravadi R and Thompson CB: The survival kinases Akt and Pim as potential pharmacological targets. J Clin Invest 115: 2618-2624, 2005.

29. Liang H, Hittelman W and Nagarajan L: Ubiquitous expression and cell cycle regulation of the protein kinase PIM-1. Arch Biochem Biophys 330: 259-265, 1996.

30. Morishita D, Katayama R, Sekimizu K, Tsuruo T and Fujita N: Pim kinases promote cell cycle progression by phosphorylating and down-regulating $\mathrm{p} 27^{\mathrm{Kip1}}$ at the transcriptional and posttranscriptional levels. Cancer Res 68: 5076-5085, 2008.

31. Block KM, Hanke NT, Maine EA and Baker AF: IL-6 stimulates STAT3 and Pim-1 kinase in pancreatic cancer cell lines. Pancreas 41: 773-781, 2012.

32. Mondello P, Cuzzocrea S and Mian M: Pim kinases in hematological malignancies: Where are we now and where are we going? J Hematol Oncol 7: 95, 2014. 